\title{
A Phase 1b Study of Vismodegib with Pirfenidone in Patients with Idiopathic Pulmonary Fibrosis
}

\author{
Antje Prasse - Murali Ramaswamy - Shaun Mohan · Lin Pan • \\ Andrew Kenwright · Margaret Neighbors · Paula Belloni · \\ Peter P. LaCamera
}

Received: April 29, 2019 / Published online: July 19, 2019

(C) The Author(s) 2019

\begin{abstract}
Introduction: Components of the hedgehog signaling pathway are upregulated in patients with idiopathic pulmonary fibrosis (IPF). Vismodegib, a small-molecule inhibitor of hedgehog signaling, when used in combination with currently available antifibrotic therapy, may be more efficacious than antifibrotics alone. The objective of this study was to evaluate the safety and tolerability of vismodegib plus pirfenidone in patients with IPF.
\end{abstract}

Enhanced Digital Features To view enhanced digital features for this article go to https://doi.org/10.6084/ m9.figshare.8332442.

Electronic supplementary material The online version of this article (https://doi.org/10.1007/s41030019-0096-8) contains supplementary material, which is available to authorized users.

\footnotetext{
A. Prasse $(\bowtie)$

Hannover Medical School and Fraunhofer Institute for Toxicology and Experimental Medicine, Hannover, Germany

e-mail: prasse.antje@mh-hannover.de

M. Ramaswamy

PulmonIx, LLC, and Cone Health, Greensboro, NC, USA

S. Mohan · L. Pan · A. Kenwright · M. Neighbors .

P. Belloni

Genentech, Inc., South San Francisco, CA, USA

P. P. LaCamera

St. Elizabeth's Medical Center, Boston, MA, USA
}

Methods: Twenty-one patients were enrolled in a phase $1 \mathrm{~b}$ open-label trial to receive vismodegib $150 \mathrm{mg}$ plus pirfenidone $2403 \mathrm{mg}$ /day once daily. Key endpoints were safety, tolerability, and pharmacokinetics. Exploratory endpoints included change from baseline to week 24 in \% predicted forced vital capacity (FVC) and University of California, San Diego Shortness of Breath Questionnaire (UCSD-SOBQ) scores, as well as pharmacodynamic changes in hedgehog biomarker C-X-C motif chemokine ligand 14 (CXCL14).

Results: All patients reported at least one treatment-emergent adverse event (AE), most frequently muscle spasms (76.2\%). Serious AEs were reported in $14.3 \%$ of patients; one event of dehydration was considered related to vismodegib. One patient died due to IPF progression, unrelated to either treatment. More patients discontinued vismodegib than pirfenidone ( $42.9 \%$ vs. $33.3 \%$, respectively). Changes from baseline to week 24 in \% predicted FVC and UCSD-SOBQ scores were within known endpoint variability. In contrast to findings in basal cell carcinoma, vismodegib had no effect on circulating CXCL14 levels.

Conclusion: The safety profile was generally consistent with the known profiles of both drugs, with no new safety signals observed in this small cohort. There was no pharmacodynamic effect on CXCL14 levels. Future development of vismodegib for IPF may be limited due to tolerability issues. 
Trial Registration: ClinicalTrials.gov NCT02648048.

Plain Language Summary: Plain language summary available for this article.

Funding: F. Hoffmann-La Roche Ltd. and Genentech, Inc.

Keywords: Hedgehog signaling pathway; Idiopathic pulmonary fibrosis; Pirfenidone; Safety; Tolerability; Vismodegib

\section{PLAIN LANGUAGE SUMMARY}

Idiopathic pulmonary fibrosis (IPF) is a progressive, irreversible, and fatal lung disease. Pirfenidone is a drug that slows disease progression in patients with IPF, but it may be more effective when combined with other drugs that target different disease pathways. This study evaluated the safety of vismodegib, an inhibitor of signaling involved in IPF, and pirfenidone in 21 patients with IPF. All patients receiving the two drugs experienced at least one side effect. The most common side effect of the two drugs was muscle spasms. Serious side effects were noted in $14 \%$ of patients, and one dehydration side effect was related to vismodegib. More patients stopped taking vismodegib than pirfenidone. Vismodegib will not be pursued as a drug for IPF because patients cannot tolerate it.

\section{INTRODUCTION}

Idiopathic pulmonary fibrosis (IPF) is a progressive, irreversible, fatal fibrotic lung disease with a median survival of 2-5 years after diagnosis $[1,2]$. Most patients with IPF are aged $>50$ years, with symptoms of progressive dyspnea and nonproductive cough [1]. The clinical course of IPF in an individual patient is challenging to predict, with a variable rate of disease progression and decline [3].

IPF has a histopathologic pattern of usual interstitial pneumonia (UIP), which is characterized by patchy fibrosis interspersed with areas of normal lung appearance and regions of dense scar tissue [1]. Ongoing disease activity is hypothesized to occur at the transition zones between normal and fibrotic lung [4, 5]. A model of IPF pathogenesis suggests that alterations to the alveolar epithelium initiates mesenchymal cell expansion and differentiation as well as production of fibrogenic factors that result in excessive extracellular matrix deposition [6]. Multiple studies have investigated the role of the hedgehog signaling pathway in the pathogenesis of fibrosis [7-9]. Hedgehog signaling regulates epithelial and mesenchymal interactions in many tissues during mammalian embryogenesis [10]. Epithelial damage and subsequent dysfunctional epithelial responses may drive aberrant fibroblast activation and differentiation during fibrosis. Hedgehog signaling appears to be a strong inducer of fibrogenic responses in vitro and tissue fibrosis in vivo [9].

Components of the hedgehog pathway are upregulated in the lungs of patients with IPF [11-15]. Hedgehog signaling can contribute to fibrogenesis and is part of the epithelial-mesenchymal cross talk involving other pathways active in IPF, such as transforming growth factor $\beta, \mathrm{C}-\mathrm{X}-\mathrm{C}$ motif chemokine receptor type 4 , and interleukin 13, resulting in increased myofibroblast differentiation, extracellular matrix production, motility, and survival. Studies comparing lung samples from patients with IPF and control individuals have demonstrated that expression of hedgehog ligands in type II alveolar and bronchiolar epithelial cells is upregulated under conditions of disease [15]. Also, expression of hedgehog target genes, such as C-X-C motif chemokine ligand 14 (CXCL14), is increased in lung fibroblasts and detected at elevated levels in lungs of patients with IPF [12, 13, 15-17].

Vismodegib, a small-molecule inhibitor of the hedgehog signaling pathway, binds to and inhibits smoothened, a transmembrane protein involved in canonical hedgehog signaling [18]. Vismodegib received approval by the US Food and Drug Administration in 2012 for the treatment of adults with metastatic basal cell carcinoma (BCC) or with locally advanced BCC that had recurred following surgery or who were not candidates for surgery or radiation therapy [19]. In the pivotal registration study (SHH4476g) in metastatic or locally advanced BCC, $30 \%$ of 
patients (95\% CI 16-49\%; $p=0.001$ ) achieved the primary efficacy outcome of an objective response as assessed by an independent review facility [18]. The median duration of treatment was 9.8 months at the time of the primary analysis [18]. Treatment-emergent adverse events (TEAEs), defined as any new AE reported or any worsening of an existing condition reported on or after the first dose of study drug, occurred at a rate of $\geq 10 \%$ and included decreased appetite, dysgeusia, ageusia, nausea, diarrhea, constipation, vomiting, alopecia, muscle spasms, amenorrhea, weight loss, and fatigue [18].

Previous reports have shown that circulating CXCL14 protein levels are significantly higher in plasma from patients with IPF than in controls, and circulating CXCL14 levels are significantly reduced upon vismodegib treatment in patients with cancer, indicating that circulating CXCL14 levels can reflect hedgehog pathway signaling in some settings [16]. However, noncanonical hedgehog signaling can also occur [14], and it is unclear whether hedgehog pathway activation in IPF is predominantly canonical. Also, there are several other pathways active in IPF with potential to contribute to CXCL14 levels, including signaling by WNT, hypoxiainducible factor 1-alpha, vascular endothelial growth factor, epidermal growth factor, and oxidative stress [17]. Therefore, CXCL14 was explored in this study to determine whether it was suitable as an indicator of pharmacological activity for vismodegib in IPF.

Pirfenidone is one of two approved oral antifibrotic therapies for the treatment of IPF [20-22]. Although its exact mechanism of action has not been fully established, pirfenidone has exhibited anti-inflammatory, antioxidant, and antifibrotic properties in some studies, including reductions in levels of tumor necrosis factor $\alpha$, transforming growth factor $\beta$, and more recently, zinc finger protein GLI2, a component of the hedgehog pathway [23-25]. Notably, these studies were not conducted using physiologically relevant concentrations of pirfenidone. Clinically, pirfenidone has slowed disease progression of IPF as measured by changes in percent predicted forced vital capacity (\% predicted FVC) and reduced the risk of death as demonstrated in pooled analyses of three phase 3 pivotal trials: ASCEND (Study 016, NCT01366209) and the CAPACITY studies (Study 004, NCT00287716 and Study 006, NCT00287729]) [20, 21, 26, 27]. In patients with IPF, pirfenidone has been generally well tolerated, with manageable AEs, most notably gastrointestinal and skin-related [20, 21, 26, 28]. Previous studies have shown that pirfenidone does not modulate CXCL14 levels in patients providing biomarker samples from these trials [29].

Targeting multiple pathways involved in the pathogenesis of lung fibrosis with combination therapy of pirfenidone and vismodegib may maximize the efficacy of the currently available standard of care for patients with IPF. The primary objective of this study was to evaluate the safety and tolerability of vismodegib in combination with pirfenidone in patients with IPF.

\section{METHODS}

\section{Study Design}

ISLAND2 was a single-arm, multicenter, openlabel, phase $1 \mathrm{~b}$ trial that assessed the safety and tolerability of vismodegib in combination with pirfenidone in patients with IPF (NCT02648048). Patients who provided written informed consent entered a screening period of up to 28 days to establish study criteria (visit 1 ) (Fig. 1). Eligible patients were then enrolled in a single treatment arm for oral administration of vismodegib $150 \mathrm{mg}$ once daily plus pirfenidone $\leq 801 \mathrm{mg} /$ day three times daily with food.

The treatment duration was 24 weeks, with the first dose of vismodegib administered at the enrollment visit (visit 2, day 1). All patients who completed therapy, as well as those who discontinued study treatment early, were asked to complete a 30-day safety follow-up period during which only pirfenidone was administered. The maximum total study duration for any patient was approximately 28 weeks from the first administration of vismodegib. No dose reductions of vismodegib were permitted based on previous studies in BCC [18]. Treatment with 


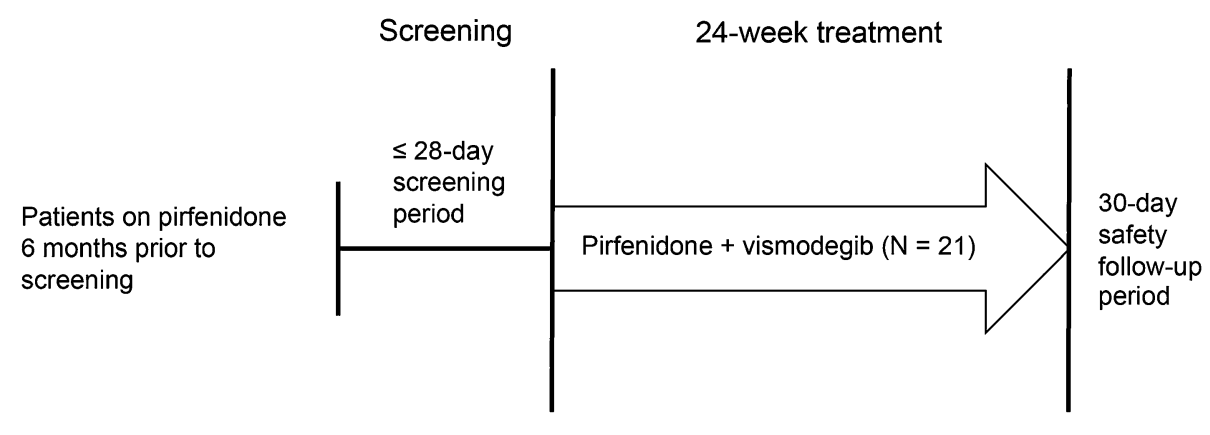

Visit $1 \quad$ Visit 2, day 1

Fig. 1 Study design

vismodegib could be interrupted for up to 8 weeks for evaluation of an intolerable toxicity; the original dose was maintained upon restart of treatment.

All procedures followed were in accordance with the ethical standards of the responsible committee on human experimentation (institutional and national) and with the Helsinki Declaration of 1964, as revised in 2013. All investigators obtained institutional review board (IRB) approval for the investigation either through the central IRB located in Seattle, WA, or via local IRBs (Table S1 in the Electronic Supplementary Material), and all patients provided informed consent. The ClinicalTrials.gov registration number for this study is NCT02648048.

\section{Patients}

Patients who were aged $40-80$ years at visit 1 , had clinical symptoms consistent with IPF for $\geq 12$ months, and had a diagnosis of UIP or IPF by high-resolution computed tomography (HRCT) and/or surgical lung biopsy within 5 years (Table S2 in the Electronic Supplementary Material) were enrolled [1]. A central review assessment of HRCT was performed during the screening period or $\leq 12$ months prior to the start of screening. Patients had to be receiving stable pirfenidone ( $\geq 1602 \mathrm{mg} /$ day) for $\geq 8$ weeks prior to randomization, without adverse drug reactions, tolerating pirfenidone 1602-2403 mg/day for $\geq 24$ weeks prior to and during screening. Additional criteria included \% predicted FVC $\geq 50 \%$ and $\leq 100 \%$ at screening; percent predicted diffusing capacity for carbon monoxide (DLco) $\geq 30 \%$ and $\leq 90 \%$ at screening; adequate hematopoietic, renal, and liver function; and absolute avoidance of pregnancy.

Key exclusion criteria were known hypersensitivity to any components of the study drugs or the drugs themselves; prior treatment with vismodegib or any hedgehog pathway inhibitor; evidence of other known causes of interstitial lung disease; hospitalization due to an IPF exacerbation $\leq 4$ weeks prior to or during screening; lung transplant expected within 6 months of screening; evidence of clinically significant lung disease other than IPF; known current malignant neoplasm or current evaluation for a potential malignancy; tobacco smoking within 3 months of screening or unwillingness to avoid smoking throughout the study; and any condition that, as assessed by the investigator, might be significantly exacerbated by the known AEs associated with pirfenidone.

\section{Assessments}

Disease-specific assessments included spirometry, DLco, HRCT, surgical lung biopsy, and University of California, San Diego Shortness of Breath Questionnaire (UCSD-SOBQ) [30, 31]. Safety assessments included TEAEs, AEs of special interest (AESIs), clinical laboratory tests, and vital signs. Vismodegib-specific AESIs included muscle spasms and drug-induced liver injury, for which monitoring was conducted for 
elevated alanine aminotransferase or aspartate aminotransferase levels, with either elevated bilirubin levels or clinical jaundice. Pirfenidonespecific AESIs included gastrointestinal and skin disorders, elevated liver enzyme values, and photosensitivity reaction or rash.

Serum and plasma samples were collected during screening, at baseline, and throughout the study for pharmacokinetics, exploratory analyses, and laboratory assessments. Single predose trough plasma samples were collected from all patients at weeks 4,12 , and 24 and at the 30-day safety follow-up visit following the last dose of vismodegib. The total and unbound concentrations of vismodegib in plasma were quantified using a validated solid-phase extraction and liquid chromatography-tandem mass spectrometry method [32].

CXCL14 was the primary hedgehog pathway biomarker investigated to evaluate vismodegib pharmacodynamic activity and was measured in plasma using a prototype Elecsys ${ }^{\circledR}$ platform (Cobas e; Roche Diagnostics, Penzberg, Germany) [16].

\section{Study Objectives}

The primary objective was to evaluate the safety and tolerability of the vismodegib and pirfenidone combination. The pharmacokinetics objective was to evaluate the pharmacokinetics of vismodegib when administered in combination with pirfenidone. The exploratory objectives were to investigate the efficacy of vismodegib in combination with pirfenidone based on change from baseline to week 24 in \% predicted FVC and in dyspnea, as measured by UCSD-SOBQ score, as well as pharmacodynamic change in CXCL14 levels.

\section{Statistical Analyses}

The analysis population comprised the safety population, intent-to-treat population (ITT), pharmacokinetics population, and pharmacodynamics population. The safety population consisted of all enrolled patients who received at least one dose of any study treatment and had at least one post-dose safety assessment.
The ITT population consisted of all enrolled patients who received at least one dose of any study treatment. The pharmacokinetics population included all safety-evaluable patients with a post-dose pharmacokinetics sample. The pharmacodynamics population included all patients with non-missing biomarker data available at baseline and at least one non-missing post-baseline measurement in addition to meeting dosing criteria (discontinued vismodegib $<3$ consecutive days prior to sample collection or redosed continuously $>11$ days after a lapse in dosing for any reason).

The study was not powered, and no hypothesis testing was performed due to the exploratory nature of the study. A sample size of approximately 20 patients was determined based on safety and clinical considerations. All data were summarized using descriptive statistics. The severity of AEs was graded according to the National Cancer Institute Common Terminology Criteria for Adverse Events version 4.0 [33]. Log-transformed biomarker data were evaluated by a linear mixed-effects model, with visit variable as a fixed effect and patient as a random effect to determine whether on-study biomarker levels at weeks 4, 12, and 24 differed from baseline, with two-sided 95\% confidence intervals.

\section{RESULTS}

\section{Patient Disposition}

A total of 31 patients with IPF receiving background pirfenidone were screened, of whom 10 were deemed ineligible (Fig. 2). Overall, 21 patients were enrolled between January 15, 2016, and May 18, 2016, at 16 sites in the United States and one site in Germany $(n=5)$. Fifteen patients (71.4\%) completed the 24 -week treatment phase and the safety follow-up period. Six patients (28.6\%) discontinued the study during the 24-week treatment period (Table 1). Four patients (19.0\%) discontinued due to AEs, one patient $(4.8 \%)$ withdrew, and one patient $(4.8 \%)$ discontinued for other reasons. Nine patients $(42.9 \%)$ stopped vismodegib treatment during the study, of whom seven (33.3\%) 


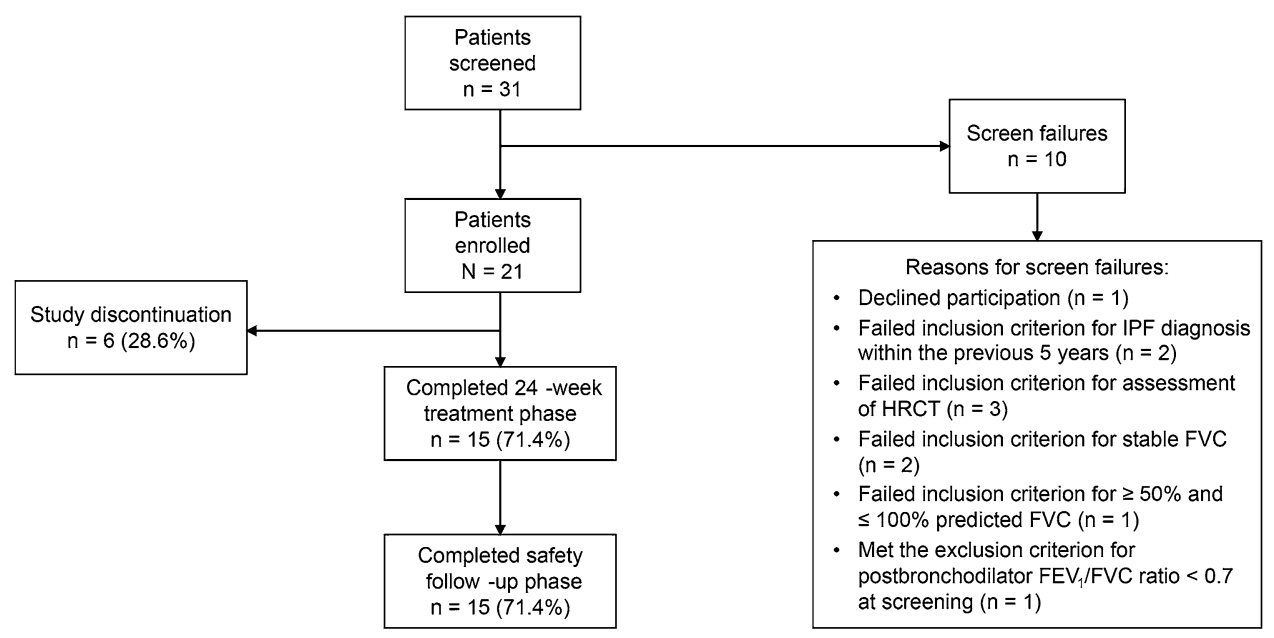

Fig. 2 Patient disposition. $F E V_{1}$ forced expiratory volume in $1 \mathrm{~s}, F V C$ forced vital capacity, $H R C T$ high-resolution computed tomography, IPF idiopathic pulmonary fibrosis

Table 1 Patient disposition through week 24

\begin{tabular}{|c|c|}
\hline Status, $n(\%)$ & $\begin{array}{l}\text { Vismodegib } \\
150 \mathrm{mg} / \text { day }+ \\
\text { pirfenidone } \leq 2403 \mathrm{mg} / \text { day } \\
(N=21)\end{array}$ \\
\hline $\begin{array}{l}\text { Completed 24-week } \\
\text { treatment phase }\end{array}$ & $15(71.4)$ \\
\hline $\begin{array}{l}\text { Completed safety follow- } \\
\text { up }\end{array}$ & $15(71.4)$ \\
\hline $\begin{array}{l}\text { Discontinued 24-week } \\
\text { treatment phase }\end{array}$ & $6(28.6)$ \\
\hline Adverse event & $4(19.0)$ \\
\hline Withdrawal by patient & $1(4.8)$ \\
\hline Other & $1(4.8)$ \\
\hline
\end{tabular}

discontinued all treatment (Table 2). The primary reason for discontinuation of study drug was a TEAE associated with vismodegib (six of nine patients) or pirfenidone (one of seven patients).

\section{Patients}

All 21 enrolled patients received at least one dose of study drug and were included in the safety and intent-to-treat populations. Twenty patients were included in the pharmacokinetics analysis population. By the end of week 24,12

Table 2 Discontinuations of vismodegib and pirfenidone through week $24(N=21)$

\begin{tabular}{lll}
\hline Status, $\boldsymbol{n}(\%)$ & $\begin{array}{l}\text { Vismodegib } \\
\mathbf{1 5 0} \mathbf{~ m g / d a y}\end{array}$ & $\begin{array}{l}\text { Pirfenidone } \mathbf{5} \mathbf{2 4 0 3} \\
\mathbf{m g} / \text { day }\end{array}$ \\
\hline $\begin{array}{lll}\text { Study drug } \\
\text { discontinuations }\end{array}$ & $9(42.9)^{\mathrm{a}}$ & $7(33.3)$ \\
AEs & $6(28.6)$ & $1(4.8)$ \\
Death & 0 & 0 \\
Other & $1(4.8)$ & $1(4.8)$ \\
$\begin{array}{l}\text { Physician } \\
\text { decision }\end{array}$ & 0 & 0 \\
$\begin{array}{l}\text { Protocol } \\
\text { violation }\end{array}$ & 0 & 0 \\
$\begin{array}{l}\text { Withdrawal by } \\
\text { patient }\end{array}$ & $2(9.5)$ & $5(23.8)$ \\
\hline
\end{tabular}

$A E$ adverse event, $S A E$ serious adverse event

${ }^{a}$ Four of nine patients discontinued vismodegib due to muscle spasm AEs, and one of nine patients discontinued vismodegib due to a dehydration SAE

patients remained in the intent-to-treat population.

The mean (standard deviation [SD]) age was 70.6 (6.8) years, and most patients were male and white $(90.5 \%$ each) (Table 3$)$. Eighteen patients (85.7\%) had a history of smoking. The mean (SD) IPF duration was 2.45 (1.37) years. 
Table 3 Baseline demographics and clinical characteristics

\begin{tabular}{|c|c|}
\hline Characteristic & $\begin{array}{l}\text { Vismodegib } 150 \mathrm{mg} / \text { day }+ \\
\text { pirfenidone } \leq 2403 \mathrm{mg} / \text { day } \\
(N=21)\end{array}$ \\
\hline Age, mean (SD), years & $70.6(6.8)$ \\
\hline Male, $n(\%)$ & $19(90.5)$ \\
\hline White, $n$ (\%) & $19(90.5)$ \\
\hline BMI, mean (SD), $\mathrm{kg} / \mathrm{m}^{2}$ & $28.93(3.27)$ \\
\hline $\begin{array}{l}\text { Time from IPF diagnosis, } \\
\text { mean (SD), years }\end{array}$ & $2.45(1.37)$ \\
\hline \multicolumn{2}{|l|}{ Smoking status, $n$ (\%) } \\
\hline Never & $3(14.3)$ \\
\hline Former & $18(85.7)$ \\
\hline Current & 0 \\
\hline $\begin{array}{l}\mathrm{FVC} \text {, mean }(\mathrm{SD}), \% \\
\text { predicted }\end{array}$ & $67.38(13.29)$ \\
\hline $\begin{array}{l}\text { DLco, mean (SD), \% } \\
\text { predicted }^{\mathrm{a}}\end{array}$ & $62.79(24.13)$ \\
\hline $\begin{array}{l}\text { UCSD-SOBQ score, } \\
\text { mean }(\mathrm{SD})\end{array}$ & $48.62(22.49)$ \\
\hline
\end{tabular}

$B M I$ body mass index, $D L C O$ diffusing capacity for carbon monoxide, $F V C$ forced vital capacity, IPF idiopathic pulmonary fibrosis, $S D$ standard deviation, UCSD-SOBQ University of California, San Diego Shortness of Breath Questionnaire

${ }^{a}$ Corrected for alveolar volume and hemoglobin

The most frequent concurrent medical conditions reported by patients were hypertension $(n=12 \quad[57.1 \%]), \quad$ hyperlipidemia $\quad(n=8$ [38.1\%]), and gastroesophageal reflux disease $(n=7$ [33.3\%]). The most commonly reported concomitant medications were proton pump inhibitors $(n=18[85.7 \%])$, salicylates $(n=13$ [61.9\%]), and statins $(n=13$ [61.9\%]). Four patients (19.0\%) reported prior pirfenidone use.

\section{Safety}

All 21 patients experienced at least one TEAE during the study, and a total of 135 TEAEs were reported (Table 4 ), most of which were considered mild or moderate in intensity by the investigator. The most frequent TEAEs were muscle spasms $(n=16$ [76.2\%]) and dysgeusia $(n=13$ [61.9\%]) (Table 5; Table S3 in the Electronic Supplementary Material). One $\mathrm{AE}$ of decreased appetite of life-threatening intensity occurred and was considered by the investigator to be related to vismodegib.

A total of five treatment-emergent serious AEs (TESAEs) were reported in three patients (one event each of parainfluenza virus infection, dehydration, and pulmonary embolism; two events of IPF progression). The TESAE of dehydration was considered related to vismodegib by the investigator (Table 4 ).

Table 4 Safety overview

\begin{tabular}{|c|c|}
\hline & $\begin{array}{l}\text { Vismodegib } 150 \mathrm{mg} / \text { day }+ \\
\text { pirfenidone } \leq 2403 \mathrm{mg} / \text { day } \\
(N=21)\end{array}$ \\
\hline $\begin{array}{l}\text { Patients with } \geq 1 \text { TEAE, } \\
\quad n(\%)\end{array}$ & $21(100)$ \\
\hline Total number of TEAEs & 135 \\
\hline $\begin{array}{l}\text { TEAE leading to } \\
\text { withdrawal from study, } \\
n(\%)\end{array}$ & $4(19.0)$ \\
\hline $\begin{array}{l}\text { Patients with } \geq 1 \text { SAE } \\
\quad n(\%)\end{array}$ & $3(14.3)$ \\
\hline Total number of SAEs & 5 \\
\hline Related SAE, $n(\%)^{\mathrm{a}}$ & $1(4.8)$ \\
\hline Deaths, $n(\%)^{\mathrm{b}}$ & $1(4.8)$ \\
\hline \multicolumn{2}{|l|}{ Patients with $\geq 1$ AESI } \\
\hline Muscle spasms, $n$ (\%) & $16(76.2)$ \\
\hline $\begin{array}{l}\text { Total number of } \\
\text { muscle spasm events }\end{array}$ & 28 \\
\hline Infections, $n(\%)$ & $7(33.3)$ \\
\hline $\begin{array}{l}\text { Total number of } \\
\text { infections }\end{array}$ & 7 \\
\hline
\end{tabular}

$A E S I$ adverse event of special interest, IPF idiopathic pulmonary fibrosis, $S A E$ serious adverse event, TEAE treatment-emergent adverse event

a SAE dehydration related to vismodegib

b Cause of death reported as IPF 
Table 5 Treatment-emergent adverse events in $\geq 10 \%$ of patients in the safety-evaluable population

\begin{tabular}{ll}
\hline TEAE, $\boldsymbol{n}$ (\%) & $\begin{array}{l}\text { Vismodegib } \mathbf{1 5 0} \mathbf{~ m g} / \text { day }+ \\
\text { pirfenidone } \leq \mathbf{2 4 0 3} \mathbf{~ m g} / \mathbf{d a y} \\
(\boldsymbol{N}=\mathbf{2 1})\end{array}$ \\
\hline Total & $21(100)$ \\
Muscle spasms & $16(76.2)$ \\
Dysgeusia & $13(61.9)$ \\
Alopecia & $7(33.3)$ \\
Weight loss & $7(33.3)$ \\
Decreased & $6(28.6)$ \\
$\quad$ appetite & $3(14.3)$ \\
IPF & $3(14.3)$ \\
Nausea &
\end{tabular}

IPF idiopathic pulmonary fibrosis, TEAE treatmentemergent adverse event

Sixteen patients $(76.2 \%)$ reported at least one AESI of muscle spasms, all considered vismodegib-related (28 total events). Most muscle spasms occurred in both arms and both legs, started early during treatment at week 4 , and were of mild or moderate intensity; however, four of nine patients who discontinued vismodegib reported reasons related to muscle spasms. Infection AESIs were reported by seven patients (33.3\%) and comprised respiratory tract infections (two patients), and one each of bronchitis, pneumonia, rhinitis, influenza, and parainfluenza. One patient died due to progression of IPF after completion of the last dose of drug administration, but this event was considered unrelated to either vismodegib or pirfenidone.

Patients received vismodegib treatment for 5-26 weeks, with 11 of 21 patients $(52 \%)$ receiving the protocol-defined 24 weeks of treatment. The mean (SD) treatment duration was 19.2 (6.6) and 22.9 (6.6) weeks for vismodegib and pirfenidone, respectively (Table S4 in the Electronic Supplementary Material). One patient had a treatment holiday (i.e., missed treatment for $\geq 7$ days) for 34 days. Another two patients had vismodegib dosing interrupted for $<7$ days.

\section{Pharmacokinetics}

The observed total and free trough plasma concentrations of vismodegib following daily oral administration of vismodegib plus pirfenidone were relatively constant at steady state over time (range 7-9 $\mu \mathrm{g} / \mathrm{mL}$ and $0.07-0.09 \mu \mathrm{g} /$ $\mathrm{mL}$, respectively) (Table S5 in the Electronic Supplementary Material). These levels were consistent with vismodegib exposures in patients with cancer in the previous studies of vismodegib using the same dosing regimen [32, 34-37].

\section{Pharmacodynamics}

CXCL14 baseline levels were comparable to those in other IPF studies (data not shown). No meaningful change from baseline was observed at weeks 4,12 , and 24 after vismodegib treatment (Fig. 3).

\section{Exploratory Efficacy}

The mean (SD) change from baseline in \% predicted FVC to week 24 was $2.0 \%$ (5.94\%). Changes in \% predicted FVC from baseline for the 12 patients in the ITT population were within the known inherent variability for this endpoint (Fig. 4) [38-40].

A positive trend in dyspnea as assessed by UCSD-SOBQ was observed in this small cohort of patients who completed the trial. The mean (SD) changes from baseline in UCSD-SOBQ score at weeks $12(n=19)$ and $24(n=12)$ were 2.00 (20.29) and -4.58 (17.05), respectively. However, due to the large number of patient discontinuations (12 patients remaining by week 24 for the exploratory analysis), the observed change was lower than the expected minimum clinically important difference of 8 in an IPF population [41].

\section{DISCUSSION}

The rationale for investigating the combination of vismodegib with pirfenidone was to increase the efficacy of currently available antifibrotic 


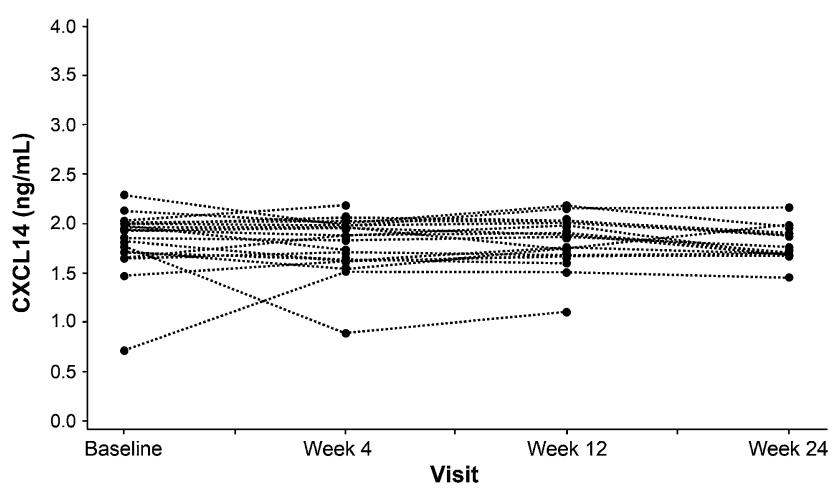

Fig. 3 Individual CXCL14 levels. Plasma CXCL14 levels in individuals over time after initiation of vismodegib treatment. Baseline is the patient's last observation prior to initiation of vismodegib. Dashed lines connect matched longitudinal samples from individual patients. CXCL14 C-X-C motif chemokine ligand 14

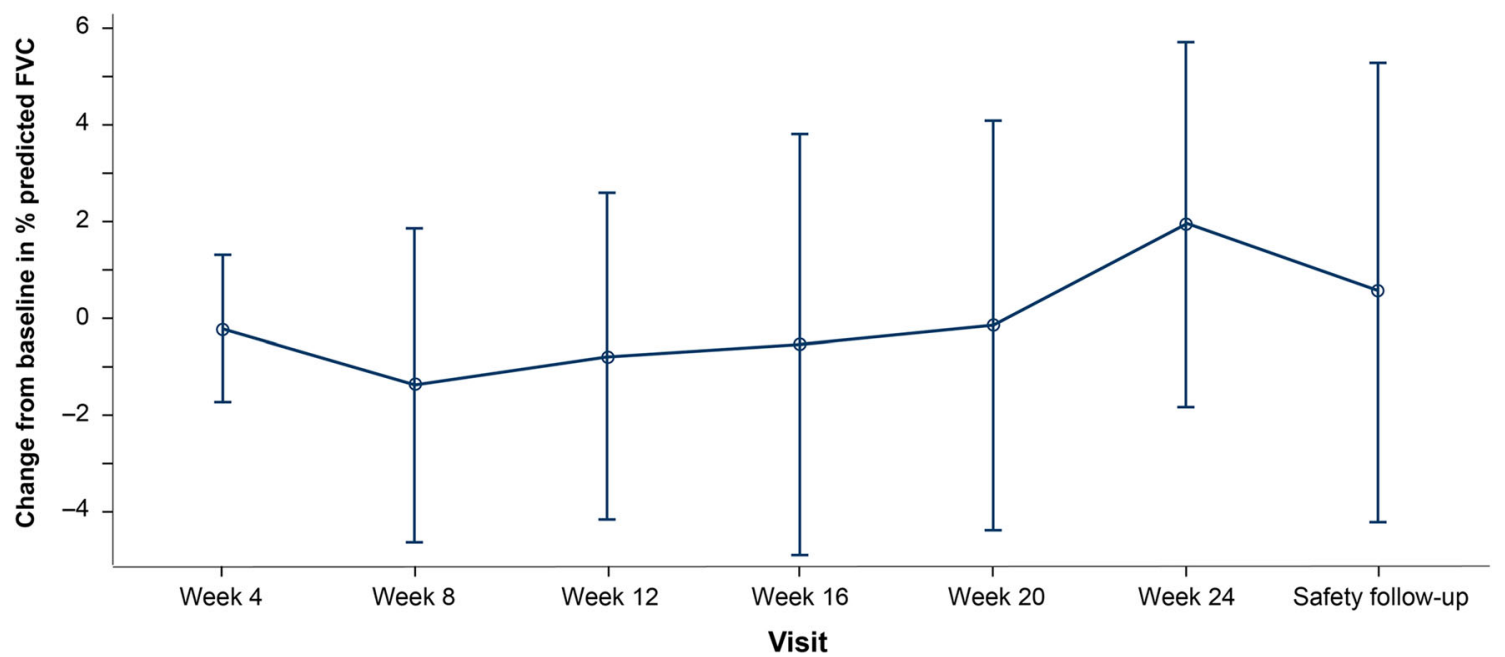

Fig. 4 Mean change from baseline \% predicted FVC score for the 12 patients in the ITT population. Within a given visit, the best (maximum) result for the parameter of interest was used if it was from an acceptable blow as assessed by the over-reader. Baseline is the patient's last

therapies in patients with IPF. In this phase $1 \mathrm{~b}$ trial, the overall safety profiles observed with both study drugs were consistent with the known safety profiles for vismodegib and pirfenidone. Rates of TEAEs based on preferred terms in the System Organ Class groups of infection as well as respiratory, thoracic, and mediastinal disorders were evenly distributed. No new safety signals were observed, and the incidence of SAEs was low. There was one death due to IPF disease progression. observation prior to initiation of vismodegib. If multiple records were collected during the same visit, then the last record within that last visit was used for the analysis. FVC forced vital capacity, ITT intent to treat

The treatment discontinuation rate for vismodegib (42.9\%) was moderately higher than that for pirfenidone (33.3\%) and higher than those observed in other pirfenidone monotherapy and combination studies $[20,21,42]$. The most frequently reported TEAEs were muscle spasms (76.2\%) and dysgeusia $(61.9 \%)$, which may have contributed to the observed elevated discontinuation rate. The rates of muscle spasms and dysgeusia were consistent with the rates observed in the phase 
$1 \mathrm{~b} / 2$ registration trials in $\mathrm{BCC}(68 \%$ and $51 \%$, respectively) [18]. This AE profile associated with vismodegib treatment is likely the mechanism based on similar AEs that have been reported for another inhibitor of hedgehog signaling, sonidegib [43]. In a phase 2 study of sonidegib, muscle spasms were the most commonly reported $\mathrm{AE}$ in $49 \%$ and $67 \%$ of patients in the $200 \mathrm{mg}$ and $800 \mathrm{mg}$ groups, respectively, and the most common $\mathrm{AE}$ resulting in treatment discontinuation [43].

The plasma concentrations of vismodegib in patients with IPF were consistent with those observed previously in oncology studies in which the drug was given in the same dosing regimen [34-37].

There is evidence from patients with cancer that plasma CXCL14 levels are sensitive to modulation by vismodegib, yet no pharmacodynamic effect on this biomarker was observed in our study of patients with IPF [16]. It is unlikely that this result is indicative of failure to engage the receptor, because the safety findings are considered to be target-related. More likely, this finding indicates that the systemic levels of this hedgehog pathway biomarker were insensitive to vismodegib and may suggest that noncanonical, smoothened-independent signaling contributes more to hedgehog pathway activity in patients with IPF. However, it could also indicate that CXCL14 is not suitable for monitoring hedgehog pathway activity in IPF because other pathways active in IPF have a greater influence on circulating CXCL14 levels.

In a 24-week study of patients with IPF treated with pirfenidone, a decrease in FVC ( $\approx 35-70 \mathrm{~mL}$ ) would have been expected [38]. In this study, however, a slight increase in FVC was observed. Furthermore, after vismodegib discontinuation ( 24 weeks), FVC values started to decrease again. These FVC changes were likely not meaningful, particularly given the small sample size and heterogeneous intraindividual variability in treatment response observed in real-world patient cohorts [39, 44]. Based on the lack of a comparator arm, short study duration, small number of patients, and the high discontinuation rate, it is difficult to draw any conclusions from these findings. However, the magnitude of the effect of combination therapy with pirfenidone and vismodegib on FVC was within a range similar to that observed in a trial that assessed combination therapy with pirfenidone and nintedanib for 6 months [42].

Limitations of the study include a small sample size, high discontinuation rate, and the lack of a comparator arm and statistical powering. The observed change from baseline in FVC and UCSD-SOBQ at week 24 were within the known variability inherent in these endpoints.

\section{CONCLUSION}

No new safety signals were identified with the combination of pirfenidone and vismodegib in patients with IPF. The development of vismodegib for treatment of patients with IPF has been discontinued due to the tolerability issues observed in this study. Future studies will be needed to determine how hedgehog signaling can be efficiently inhibited with fewer systemic AEs (e.g., different target or mode of administration).

\section{ACKNOWLEDGEMENTS}

We thank the participants of the study. We acknowledge Astrid Scalori for study protocol development and execution as lead medical monitor of the study.

Funding. This study was funded by F. Hoffmann-La Roche Ltd. and Genentech, Inc. (South San Francisco, CA, USA). The sponsor was responsible for the clinical operations oversight, data management, medical monitoring, drug supply, statistical analysis, drug safety process, medical writing, and journal article processing charges. All authors had full access to all of the data in this study and take complete responsibility for the integrity of the data and accuracy of the data analysis.

Authorship. All named authors meet the International Committee of Medical Journal Editors (ICMJE) criteria for authorship for this article, take responsibility for the integrity of 
the work as a whole, and have given their approval for this version to be published.

Medical Writing, Editorial, and Other Assistance. Support for third-party writing assistance for this manuscript, furnished by writer Christine Gould, PhD, CMPP, of Health Interactions, Inc., was provided by $\mathrm{F}$. Hoffmann-La Roche Ltd. and Genentech, Inc.

Disclosures. Antje Prasse has received fees from Genentech, Inc., for participation as a study center in the ISLAND2 clinical trial; personal fees from Roche and Novartis; grants and personal fees from Boehringer Ingelheim, AstraZeneca, and Nitto; grants from AdAlta; and non-financial support from Roche, Boehringer Ingelheim, AstraZeneca, and Novartis. Murali Ramaswamy has received personal fees from Genentech, Inc., and the France Foundation. Shaun Mohan is an employee of Genentech, Inc. Lin Pan is an employee of Genentech, Inc. Andrew Kenwright is an employee of Genentech, Inc. Margaret Neighbors is an employee of Genentech, Inc. Paula Belloni is an employee of Genentech, Inc. Peter P. LaCamera has received personal fees for consulting and advisory boards from Genentech, Inc.

Compliance with Ethics Guidelines. All procedures performed in studies involving human participants were in accordance with the ethical standards of the institutional and/or national research committee and with the 1964 Helsinki declaration and its later amendments or comparable ethical standards. Approvals were obtained either through the central IRB in Seattle, WA, or local IRBs (see Table S1 in the Electronic Supplementary Material). Informed consent was obtained from all individual participants included in the study.

Data Availability. The data sets generated during and/or analyzed during the current study are available from the corresponding author on reasonable request.

Qualified researchers may request access to individual patient-level data through the ClinicalStudyDataRequest platform (www.clinicalstudydatarequest.com). Further details on
Roche's criteria for eligible studies are available here (https://clinicalstudydatarequest.com/ Study-Sponsors/Study-Sponsors-Roche.aspx). For further details on Roche's Global Policy on the Sharing of Clinical Information and how to request access to related clinical study documents, see here (https://www.roche.com/research_and_development/who_we_are_how_we_ work/clinical_trials/our_commitment_to_data_ sharing.htm).

Open Access. This article is distributed under the terms of the Creative Commons Attribution 4.0 International License (http:// creativecommons.org/licenses/by/4.0/), which permits unrestricted use, distribution, and reproduction in any medium, provided you give appropriate credit to the original author(s) and the source, provide a link to the Creative Commons license, and indicate if changes were made.

\section{REFERENCES}

1. Raghu G, Collard HR, Egan JJ, et al. An official ATS/ ERS/JRS/ALAT statement: idiopathic pulmonary fibrosis: evidence-based guidelines for diagnosis and management. Am J Respir Crit Care Med. 2011;183(6):788-824.

2. Nathan SD, Shlobin OA, Weir N, et al. Long-term course and prognosis of idiopathic pulmonary fibrosis in the new millennium. Chest. 2011;140(1):221-9.

3. Ley B, Collard HR, King TE Jr. Clinical course and prediction of survival in idiopathic pulmonary fibrosis. Am J Respir Crit Care Med. 2011;183(4):431-40.

4. Dempsey OJ, Kerr KM, Gomersall L, Remmen H, Currie GP. Idiopathic pulmonary fibrosis: an update. QJM. 2006;99(10):643-54.

5. Streiter RM. What differentiates normal lung repair and fibrosis? inflammation, resolution of repair, and fibrosis. Proc Am Thorac Soc. 2008;5:305-10.

6. Sakai N, Tager AM. Fibrosis of two: epithelial cellfibroblast interactions in pulmonary fibrosis. Biochim Biophys Acta. 2013;1832(7):911-21. 
7. Choi SS, Omenetti A, Syn WK, Diehl AM. The role of hedgehog signaling in fibrogenic liver repair. Int J Biochem Cell Biol. 2011;43(2):238-44.

8. Beyer C, Distler JH. Morphogen pathways in systemic sclerosis. Curr Rheumatol Rep. 2013;15(1):299.

9. $\mathrm{Hu} \mathrm{L}$, Lin $\mathrm{X}, \mathrm{Lu} \mathrm{H}$, Chen B, Bai Y. An overview of hedgehog signaling in fibrosis. Mol Pharmacol. 2015;87(2):174-82.

10. Ingham PW, McMahon AP. Hedgehog signaling in animal development: paradigms and principles. Genes Dev. 2011;15:3059-87.

11. Stewart GA, Hoyne GF, Ahmad SA, et al. Expression of the developmental sonic hedgehog (Shh) signalling pathway is up-regulated in chronic lung fibrosis and the Shh receptor patched 1 is present in circulating $\mathrm{T}$ lymphocytes. J Pathol. 2003;199(4):488-95.

12. Coon DR, Roberts DJ, Loscertales M, Kradin R. Differential epithelial expression of SHH and FOXF1 in usual and nonspecific interstitial pneumonia. Exp Mol Pathol. 2006;80(2):119-23.

13. Fitch PM, Howie SE, Wallace WA. Oxidative damage and TGF-beta differentially induce lung epithelial cell sonic hedgehog and tenascin-C expression: implications for the regulation of lung remodelling in idiopathic interstitial lung disease. Int J Exp Pathol. 2011;92(1):8-17.

14. Cigna N, Farrokhi Moshai E, Brayer S, et al. The hedgehog system machinery controls transforming growth factor-beta-dependent myofibroblastic differentiation in humans: involvement in idiopathic pulmonary fibrosis. Am J Pathol. 2012;181(6):2126-37.

15. Bolanos AL, Milla CM, Lira JC, et al. Role of sonic hedgehog in idiopathic pulmonary fibrosis. Am J Physiol Lung Cell Mol Physiol. 2012;303(11):L978-90.

16. Jia G, Chandriani S, Abbas AR, et al. CXCL14 is a candidate biomarker for hedgehog signalling in idiopathic pulmonary fibrosis. Thorax. 2017;72(9):780-7.

17. Rodriguez LR, Emblom-Callahan M, Chhina M, et al. Global gene expression analysis in an in vitro fibroblast model of idiopathic pulmonary fibrosis reveals potential role for CXCL14/CXCR1. Sci Rep. 2018;8(1):3983.

18. Sekulic A, Migden MR, Oro AE, et al. Efficacy and safety of vismodegib in advanced basal-cell carcinoma. N Engl J Med. 2012;366(23):2171-9.
19. Genentech I. Erivedge (vismodegib) capsule, for oral use [package insert]. CA: South San Francisco; 2012.

20. Noble PW, Albera C, Bradford WZ, et al. Pirfenidone in patients with idiopathic pulmonary fibrosis (CAPACITY): two randomised trials. Lancet. 2011;377(9779):1760-9.

21. King TE Jr, Bradford WZ, Castro-Bernardini S, et al. A phase 3 trial of pirfenidone in patients with idiopathic pulmonary fibrosis. $\mathrm{N}$ Engl J Med. 2014;370(22):2083-92.

22. Genentech I. Esbriet (pirfenidone) capsules and film-coated tablets, for oral use [package insert]. CA: South San Francisco; 2017.

23. Schaefer CJ, Ruhrmund DW, Pan L, Seiwert SD, Kossen K. Antifibrotic activities of pirfenidone in animal models. Eur Respir Rev. 2011;20(120):85-97.

24. Didiasova M, Singh R, Wilhelm J, et al. Pirfenidone exerts antifibrotic effects through inhibition of GLI transcription factors. FASEB J. 2017;31(5):1916-28.

25. Knuppel L, Ishikawa Y, Aichler M, et al. A novel antifibrotic mechanism of nintedanib and pirfenidone. Inhibition of collagen fibril assembly. Am J Respir Cell Mol Biol. 2017;57(1):77-90.

26. Noble PW, Albera C, Bradford WZ, et al. Pirfenidone for idiopathic pulmonary fibrosis: analysis of pooled data from three multinational phase 3 trials. Eur Respir J. 2016;47(1):243-53.

27. Nathan SD, Albera C, Bradford WZ, et al. Effect of pirfenidone on mortality: pooled analyses and meta-analyses of clinical trials in idiopathic pulmonary fibrosis. Lancet Respir Med. 2017;5(1):33-41.

28. Lancaster L, Albera C, Bradford WZ, et al. Safety of pirfenidone in patients with idiopathic pulmonary fibrosis: integrated analysis of cumulative data from 5 clinical trials. BMJ Open Respir Res. 2016;3(1):e000105 2015-000105. eCollection 2016.

29. Neighbors M, Cabanski CR, Ramalingam TR, et al. Prognostic and predictive biomarkers for patients with idiopathic pulmonary fibrosis treated with pirfenidone: post hoc assessment of the CAPACITY and ASCEND trials. Lancet Respir Med. 2018;6(8):615-26.

30. Miller MR, Hankinson J, Brusasco V, et al. Standardisation of spirometry. Eur Respir J. $2005 ; 26(2): 319-38$.

31. Graham BL, Brusasco V, Burgos F, et al. 2017 ERS/ ATS standards for single-breath carbon monoxide 
uptake in the lung. Eur Respir J. 2017;49(1):1600016. https://doi.org/10.1183/ 13993003.00016,2016 Print 2017 Jan.

32. Ding X, Chou B, Graham RA, et al. Determination of GDC-0449, a small-molecule inhibitor of the hedgehog signaling pathway, in human plasma by solid phase extraction-liquid chromatographictandem mass spectrometry. J Chromatogr B Analyt Technol Biomed Life Sci. 2010;878(9-10):785-90.

33. National Institutes of Health, National Cancer Institute. Common Terminology Criteria for Adverse Events (CTCAE) version 4.0. 2010; Available at: http://www.hrc.govt.nz/sites/default/files/ CTCAE\%20manual\%20-\%20DMCC.pdf. Accessed March 8, 2017.

34. Abou-Alfa GK, Lewis LD, LoRusso P, et al. Pharmacokinetics and safety of vismodegib in patients with advanced solid malignancies and hepatic impairment. Cancer Chemother Pharmacol. 2017;80(1):29-36.

35. Sharma MR, Karrison TG, Kell B, et al. Evaluation of food effect on pharmacokinetics of vismodegib in advanced solid tumor patients. Clin Cancer Res. 2013;19(11):3059-67.

36. Graham RA, Hop CE, Borin MT, et al. Single and multiple dose intravenous and oral pharmacokinetics of the hedgehog pathway inhibitor vismodegib in healthy female subjects. $\mathrm{Br} \mathrm{J}$ Clin Pharmacol. 2012;74(5):788-96.

37. Graham RA, Lum BL, Cheeti S, et al. Pharmacokinetics of hedgehog pathway inhibitor vismodegib (GDC-0449) in patients with locally advanced or metastatic solid tumors: the role of alpha-1-acid glycoprotein binding. Clin Cancer Res. 2011;17(8):2512-20.
38. Behr J, Bendstrup E, Crestani B, et al. Safety and tolerability of acetylcysteine and pirfenidone combination therapy in idiopathic pulmonary fibrosis: a randomised, double-blind, placebo-controlled, phase 2 trial. Lancet Respir Med. 2016;4(6):445-53.

39. Loeh B, Drakopanagiotakis F, Bandelli GP, et al. Intraindividual response to treatment with pirfenidone in idiopathic pulmonary fibrosis. Am J Respir Crit Care Med. 2015;191(1):110-3.

40. du Bois RM, Weycker D, Albera C, et al. Forced vital capacity in patients with idiopathic pulmonary fibrosis: test properties and minimal clinically important difference. Am J Respir Crit Care Med. 2011;184(12):1382-9.

41. Swigris JJ, Han M, Vij R, et al. The UCSD shortness of breath questionnaire has longitudinal construct validity in idiopathic pulmonary fibrosis. Respir Med. 2012;106(10):1447-55.

42. Flaherty KR, Sussman R, Pesci A, et al. Safety of the combined use of pirfenidone and nintedanib in patients with idiopathic pulmonary fibrosis (IPF): results from an interim analysis after 12 weeks. Am J Respir Crit Care Med. 2017;195:A5398.

43. Migden MR, Guminski A, Gutzmer R, et al. Treatment with two different doses of sonidegib in patients with locally advanced or metastatic basal cell carcinoma (BOLT): a multicentre, randomised, double-blind phase 2 trial. Lancet Oncol. 2015;16(6):716-28.

44. Biondini D, Balestro E, Lacedonia D, et al. Pretreatment rate of decay in forced vital capacity predicts long-term response to pirfenidone in patients with idiopathic pulmonary fibrosis. Sci Rep. 2018;8(1):5961. 